\title{
Editorial:
}

\section{How Safe Are Cognitively Impaired Seniors Who Live Alone?}

As the population ages, there are two important trends that have begun to converge in industrialized nations: there are more older people who live alone and there are more people with Alzheimer's disease and related dementias (Webber, Fox, \& Burnette, 1994; Chappell, 1991). The Canadian Study of Health and Aging (CSHA) (Canadian Study of Health and Aging, 1994) reported that 29 per cent of non-institutionalized Canadians with dementia live alone. Those with cognitive impairment or dementia who live alone are more frequently placed in residential care facilities than those who reside with another person. Family caregivers' decisions to institutionalize their elders are often based on their own difficulty coping with caregiving and their appraisal of their elders' needs for more care. In fact, characteristics of the caregiver are better predictors of institutional placement than are characteristics of the elder (Colerick \& George, 1986; Gold, Reis, Markiewicz, \& Andres, 1995). This situation is compounded by the fact that community support agencies cannot predict those most likely to experience significant harm so that they can focus their limited resources on those at greatest risk. Thus, cognitively impaired elderly people, who want to remain in their own home, may be inappropriately or prematurely institutionalized because of difficulties anticipated by concerned relatives and because they receive insufficient assistance from community agencies.

The CSHA (Ostbye \& Crosse, 1994) estimated that the total net cost of dementia in Canada is over $\$ 3.9$ billion per year. Per patient annual costs for those in the community are $\$ 8,096$ (for both paid and unpaid services), and for those in nursing home facilities, $\$ 28,100$. Clearly, it is more economical to maintain people in the community. At the same time, there are risks (and consequent economic costs) to leaving cognitively impaired individuals in the community. Such risks include the potential for significant harm or death due to medication errors, malnourishment, dehydration, fires and other serious consequences.

Health care professionals' greatest concern about their patients with cognitive impairment who live alone is for their safety. Many professionals experience a conflict between wanting their patients to be in a safe environment (such as an institution) and wanting to respect their rights to live where they choose. Given the importance and prevalence of this problem, one would anticipate ample literature describing the nature and frequency of difficulties experienced by these elders in the community. Yet, the only area explored in this context is driving and the risk of automobile crashes. These studies are inconclusive: some have shown increased risk of crashes with AD (Trobe, Waller, Cook-Flannagan, Teshima, \& Bieliauskas, 1996) 
and others not (Drachman, Swearer, \& Collaborative Study Group, 1993). However, automobile crashes are relatively rare events and there has been no investigation of other harmful outcomes as they relate to the cognitive impairment of the older person who lives in the community. What has been examined extensively is the outcome of institutionalization.

In studies designed to predict institutionalization, predictor models are based on caregivers' well-being/ burden or the physical or cognitive status of the patient. For example, Colerick and George (1986) reported that being married was one of the most important predictors of whether a person with $\mathrm{AD}$ was institutionalized one year later. Those in the study who had a stressed nonspousal caregiver (usually a daughter who was employed) were the most likely to be institutionalized whatever the elder's physical condition. These important findings have been confirmed subsequently in several investigations. Interestingly, the use of community services has not been found to be a major factor in predicting institutionalization (Cohen et al., 1993; McFall \& Miller, 1992; Newman, Struyk, Wright, \& Rice, 1990). Only one study (Webber et al., 1994) compared community service utilization by persons with $\mathrm{AD}$ who lived alone vs. those who resided with another. This study reported that persons with $\mathrm{AD}$ rarely sought assistance and that it was more likely to be sought by the caregivers. Also, the type of service use was different in the two groups: $\mathrm{AD}$ patients who lived alone made more use of meals, homemakers, case management and nonfamilial caregivers, whereas those who lived with others saw physicians more and made more use of day care. It would appear that community services meet different needs for the caregivers than they do for the elder recipient.The major difference between the two groups in primary caregiver characteristics was that those who lived alone were far less likely to have a spouse as their primary caregiver. Interestingly, those living alone were also less likely to have a daughter as caregiver. If one considers these findings together with those of Colerick and George (1986), the following pattern seems to be emerging. Cognitively impaired seniors with a spousal caregiver have the highest likelihood of remaining at home. After the death of their spouse, however, those who live alone who have family caregivers may be more likely to be placed in institutions than those who do not have family caregivers. Family caregivers' concerns about their relatives as well as their own stresses are the major precipitating factors leading to institutionalization.

What does the research and legislation pertaining to mental incompetency or incapacity tell us about the prediction of safety in individuals with cognitive impairment? In general, people are considered capable or competent to decide about a particular matter if they can understand the matter for which a decision is required, and appreciate the consequences of the decision. There is a presumption of capability until sufficient evidence of incapacity is advanced to refute the presumption. People are declared incapable so that someone can be appointed substitute decision-maker ${ }^{1}$ and thereby provide the necessary assistance to ensure their financial and 
personal needs are met. Although legislation varies from province to province, the general approach is that the person alleging incapacity applies to the court requesting that the individual be declared incapable, and that the court name the applicant substitute decision-maker. Usually the court will also require evidence of the incapacity from one or more health care practitioners. Most incompetency laws differentiate between capacity to manage financial affairs and capacity to make personal decisions. In Ontario, the Substitute Decisions Act provides a set of procedures and criteria to be used in the assessment and determination of legal incapacity. A designated competency assessor must make a judgement based on observation and information from an informant regarding the person's capacity to care for his/herself. This approach rather than formal assessment is used because there are no scales available that predict whether an individual is likely to experience harm due to cognitive impairment, in either the domains of personal or financial care.

The validity of existing mental competency scales has been established by comparing performance of people requiring different levels of supervision on these scales (D'Andrea, Goldberg, Searight, Gilner, \& Katz, 1991), correlating performance on these scales with caregiver ratings of behaviour, and the decisions of multidisciplinary teams (Rutman \& Silberfeld, 1992). The limitation with these approaches to validation is that none have incorporated what would be considered "gold standard" indexes of competence. The actual occurrence of acts resulting in harm to person or loss of property has not been used to validate any of these scales. The development of a scale to determine the competence of an individual based on harmful or safe outcome will represent a major clinical and research contribution to the area of mental competency.

What is most important to know about older cognitively impaired persons and their environment in order to predict whether they would experience harm? Based on previous research, we think the following might be the most important predictors of harm in these individuals: (1) the etiology of their cognitive impairment; (2) the kinds of cognitive deficits they have; (3) the behaviour disorders they display; and (4) their existing social resources. We will briefly review our rationale for identifying these four factors as critical in the prediction of harm.

Regarding the etiology of cognitive impairment, the diagnosis of $\mathrm{AD}$, for example, may be considered a high risk factor for harm because it is a progressive disease resulting in increasingly less self-awareness and capacity to learn. Previous studies (Tierney, Szalai, Snow, et al, 1996; Tierney, Szalai, Snow, \& Fisher, 1996; Jacobs et al., 1995) have demonstrated that we can predict the development and progression of $\mathrm{AD}$; however there are no studies that have examined whether $\mathrm{AD}$ patients are more vulnerable to harm at different stages of the disease than patients with other kinds of cognitive impairment. To conclude that those with a diagnosis of $\mathrm{AD}$ will experience more harm than those with other forms of cognitive impairment may be presumptive for the following reasons. First, $\mathrm{AD}$ has a variable 
course and thus individuals at various stages of the disease may differ in their ability to avoid harm. Second, people with $A D$ may be less likely to engage in activities that would cause harm than people with other forms of cognitive impairment who are more cognitively intact. For example, someone with $\mathrm{AD}$ may never decide to use the basement stairs because he/she does not remember what is stored there. However, the individual who remembers what is in the basement may impulsively attempt to retrieve what is wanted without going through the proper sequence of activities to avoid injury, i.e. turning the lights on, holding the handrail, taking one step at a time, removing obstacles, etc.

Because we do not know whether a particular dementia diagnosis heralds emergent harm, or whether the stage of the disease or existence of specific cognitive deficits is most predictive, we consider the person's cognitive abilities as a second important predictor of harm. An individual's experience of harm may be due to the presence of specific cognitive deficits or a specific combination of deficits. Cognitive abilities considered important to safe functioning in the community include memory, language, attention and concentration, reasoning, problem-solving, planning, sequencing, and executive function.

The extent to which the elder exhibits various behaviours may also be important in the prediction of significant harm. Behavioural characteristics and functional abilities considered important predictors of harm are psychotic behaviours (including delusions and hallucinations), difficulties in self-care activities of daily living (e.g., bathing), and in instrumental activities of daily living (e.g., using the telephone), acting-out behaviours, withdrawal, and wandering.

The degree to which dementia diagnosis, cognitive function and behavioural characteristics predict the experience of harm may depend on the degree of social support and assistance the individual receives. Social resources include both unpaid assistance (e.g., assistance received from relatives, friends and neighbours), and paid assistance (e.g., homemaking and professional help). The relationship between social support and the occurrence of harm is not expected to be a simple one because those receiving the most support may be receiving it because they are perceived at greatest risk but, consequently, their risk of harm may be reduced by the presence of the support. Clearly, the relationship between cognitive impairment, behavioural characteristics, dementia diagnoses, social support and the experience of harm is complex but we must begin to unravel this complexity in order to make empirically-based decisions about care. ${ }^{2}$

\section{Conclusion}

Apart from studies examining the crash rates of cognitively impaired automobile drivers, there have been no studies specifically examining the problems that cognitively impaired elders experience because of their cognitive impairment. Judgements are made daily by physicians, psychologists, 
social workers, occupational therapists, and family members about whether a cognitively impaired elder can manage to live alone. Yet these decisions are made without a solid empirical foundation for predicting harm or safety.

We do not know whether a diagnosis of dementia is predictive of significant harm or whether the stage of the dementia or the profile of cognitive abilities is more useful for prediction. Knowledge of the kinds of behaviours the individual displays, e.g., wandering, hallucinations, etc. may also be critical for accurate prediction. Furthermore, the existing informal and formal support network of the individual would be expected to moderate the influence of diagnostic, cognitive and behavioural characteristics on the experience of harm. Continued efforts in many centres to investigate this problem are necessary as Canadian demographic and economic patterns change and we are faced with the challenge of providing the highest quality of care to our seniors in a way that meets their needs for independence.

\section{Notes}

1 In Ontario, the person who is appointed to manage the affairs of a mentally incompetent person is referred to as a substitute decision-maker. However, in other Canadian jurisdictions, terms such as guardian, trustee or committee of estate are used to describe this person.

2 At Sunnybrook Health Science Centre, we are currently addressing this problem with a longitudinal, prospective study of over 200 cognitively impaired seniors who live alone. We are initially assessing each participant for dementia diagnosis, cognitive, behavioural and social characteristics. Subsequent to this initial assessment, we are following each participant for an 18-month period and recording any incident of significant harm during this period. We want to determine whether we can predict those who will experience significant harm. If accurate prediction is possible. our goal is to develop a tool that can be used by health care professionals and community agencies to target their limited resources to the neediest individuals. The Sunnybrook team of investigators include Drs. M.C. Tierney, Jocelyn Charles, Susan Jaglal, John P. Szalai, Rory H. Fisher, and W. Gary Snow. This research is supported by the Ontario Mental Health Foundation, Alzheimer Association (U.S.), Carolyn Sifton Foundation and Alva Foundation.

\section{References}

Canadian Study of Health and Aging. (1994). Canadian study of health and aging: Study methods and prevalence of dementia. Canadian Medical Association, 150, 899-913.

Chappell, N.L. (1991). Living arrangements and sources of caregiving. Journal of Gerontology, 46, 1-8.

Cohen, C.A., Gold, D.P., Shulman, K.L., Wortley, J.T., McDonald, G., \& Wargon, M. (1993). Factors determining the decision to institutionalize dementing individuals: A prospective study. The Gerontologist, 33, 714-720.

Colerick, E.J., \& George, L.K. (1986). Predictors of institutionalization among caregivers of patients with Alzheimer's disease. Journal of the American Geriatrics Society, 34, 493-498.

D'Andrea, J., Goldberg, M.A., Searight, H.R., Gilner, F., \& Katz, B. (1991). The Community Competence Scale - Short Form: A competency-based measure for 
determining residential placement of geriatric patients. Clinical Gerontologist, $10,3-10$.

Drachman, D., Swearer, J., \& Collaborative Study Group. (1993). Driving and Alzheimer's disease: The risk of crashes. Neurology, 43, 2448-2456.

Gold, D.P., Reis, M.F., Markiewicz, D., \& Andres, D. (1995). When home caregiving ends: A longitudinal study of outcomes for caregivers of relatives with dementia. Journal of the American Geriatrics Society, 43, 10-16.

Jacobs, D.M., Sano, M., Dooneief, G., Marder, K., Bell, K., \& Stern, Y. (1995). Neuropsychological detection and characterization of preclinical Alzheimer's disease. Neurology, 45, 957-962.

McFall, S., \& Miller, B.H. (1992). Caregiver burden and nursing home admission of frail elderly persons. Journal of Gerontology, 47, 73-79.

Newman, S.J., Struyk, R., Wright, P., \& Rice, M. (1990). Overwhelming odds: Caregiving and the risk of institutionalization. Journal of Gerontology, 45, 173-183.

Ostbye, T., \& Crosse, E. (1994). Net economic costs of dementia in Canada. Canadian Medical Association, 151, 1457-1464.

Rutman, D., \& Silberfeld, M. (1992). A preliminary report on the discrepency between clinical and test evaluations of competence. Canadian Journal of Psychiatry, 37, 634-639.

Tierney, M.C., Szalai, J.P., Snow, W.G., Fisher, R.H., Nores, A., Nadon, G., Dunn, E., \& St.George-Hyslop, P.H. (1996). Prediction of probable Alzheimer's disease in memory-impaired patients: A prospective longitudinal study. Neurology, 46, 661-665.

Tierney, M.C., Szalai, J.P., Snow, W.G., \& Fisher, R.H. (1996). The prediction of Alzheimer's disease: The role of patient and informant perceptions of cognitive deficits. Arch Neurol, 53, 423-427.

Trobe, J.D., Waller, P.F., Cook-Flannagan, C.A., Teshima, S.M., \& Bieliauskas, L.A. (1996). Crashes and violations among drivers with Alzheimer's disease. Arch Neurol, 53, 411-416.

Webber, P.A., Fox, P., \& Burnette, D. (1994). Living alone with Alzheimer's disease: Effects on health and social service utilization patterns. The Gerontologist, 34, 88-14.

Mary C. Tierney 